inadequate response to standard of care (SOC) therapy (NCT04058028).

Methods In this adaptive, phase 2, placebo-controlled, doseranging study, subjects $(\mathrm{N} \sim 300$, age $18-75$ years) will be randomized to receive placebo or 1 of 3 doses of AMG 570 Q2W for 52 weeks, followed by 16 weeks of safety followup. The primary objective is to evaluate efficacy of AMG 570 compared with placebo at week 24 using the SLE Responder Index (SRI-4). Key secondary endpoints include SRI-4 at week 52 with oral corticosteroid (OCS) reduction $(\geq 10$ $\mathrm{mg} /$ day at baseline to $\leq 7.5 \mathrm{mg} /$ day in weeks 44-52) and SRI4 and Lupus Low Disease Activity State at week 52. Subjects will undergo 2 screening visits to fulfill criteria for active SLE and demonstrate adherence to prior SLE treatment including OCS, immunosuppressants, and/or immunomodulators. Blood screening tests will confirm detectable serum drug levels of baseline SOC medications. RAR aims to allocate more subjects to more efficacious doses while maintaining the placebo allocation constant; the randomization ratio could be adapted after interim analyses based on clinical efficacy. The trial includes interim analyses for futility using the Bayesian approach.

Results Study ongoing.

Conclusion This study will provide safety and efficacy data for AMG 570 compared with placebo, and its adaptive trial design aims to optimize development of a novel therapy for SLE patients with inadequate response to current SOC. Acknowledgments Amgen Inc. sponsored this study.

\section{P133 COMPUTATIONAL DISCOVERY AND PRECLINICAL VALIDATION OF THERAPEUTIC LEADS WITH NOVEL MOAS FOR SYSTEMIC LUPUS ERYTHEMATOSUS (SLE)}

Isaac Hakim, Sana Mujahid, Aaron C Daugherty, Timothy S Heuer. twXAR Inc, Mountain View, CA, USA

\subsection{6/lupus-2020-eurolupus. 177}

Background Lupus is a heterogeneous, systemic disease that affects millions of patients globally with a high unmet medical need. We present results from our powerful and efficient computational drug discovery platform that identifies hits with first-in-class mechanisms of action that can advance rapidly and successfully through preclinical validation studies. The twoXAR discovery platform uses an artificial-intelligence framework to integrate diverse patient-derived biomedical data sets to build holistic and unbiased models of human disease biology. The utilization of diverse, proprietary algorithms and deep learning principles provides a highly sensitive platform to elucidate complex disease-specific associations between biology and biomedical data that are integrated with a library of existing drug molecules. This enables the identification of novel, high-value drug discovery hits with known pharmacological properties. The twoXAR platform also preserves interpretable data-driven links to disease biology to facilitate efficient validation and optimization studies.

Methods Using clinical SLE patient data, we employed the twoXAR platform to build an in-silico SLE disease model. Nine molecules with novel mechanisms of action (not previously tested as candidate clinical therapies for lupus) were identified as drug discovery hits and then characterized in preclinical efficacy studies using the MRL mouse model of lupus.
Results In preclinical validation studies with the MRL mouse model, 2 compounds were differentiated by significant efficacy and excellent tolerability. TXR-711 and TXR-712 increased renal function, decreased renal inflammation and decreased inflammation compared to vehicle-treated control mice. In particular, TXR-711 and TXR-712 significantly decreased serum blood urea nitrogen (BUN) levels, decreased proteinuria levels, and significantly improved kidney histology readouts such as glomerulonephritis and tubule basophilia. Additionally, TXR711 and TXR-712 treatment resulted in significantly decreased inguinal lymph node weight.

Conclusions TXR-711 and TXR-712 were identified as SLE drug discovery leads with novel MOAs for further preclinical development. Ongoing studies with TXR-711 and TXR-712 includes pharmacokinetic, pharmacodynamic, and additional MRL mouse efficacy characterization.

\section{P134 A PRECLINICAL DOUBLE-BLINDED, RANDOMIZED, CONTROLLED, MULTICENTER TRIAL (PRCT) ON JAK1/ JAK2 INHIBITION IN LUPUS NEPHRITIS}

\begin{abstract}
${ }^{1}$ Yutian Lei, ${ }^{2}$ Bettina Sehnert, ${ }^{2}$ Reinhard E Voll, ${ }^{3}$ Conxita Jacobs-Cachá, ${ }^{3}$ Maria Jose Soler, ${ }^{4}$ Maria D Sanchez-Niño, ${ }^{4}$ Alberto Ortiz, ${ }^{5}$ Roman D Bülow, ${ }^{5}$ Peter Boor, ${ }^{1}$ HansJoachim Anders. 'Division of Nephrology, Dept. of Medicine IV, University Hospital, LMU Munich, Munich; ${ }^{2}$ Dept. of Rheumatology and Clinical Immunology, Medical CenterUniversity of Freiburg, Faculty of Medicine, University of Freiburg, Freiburg, Germany; ${ }^{3}$ Nephrology Dept., Hospital Universitari Vall d'Hebron, Universitat Autònoma de Barcelona, Nephrology Research Group, Vall d'Hebron Research Institute (VHIR) and REDINREN Barcelona; ${ }^{4}$ IS-Fundacion Jimenez Diaz, School of Medicine, Universidad Autonoma de Madrid, Fundacion Renal Iñigo Alvarez de Toledo-IRSIN and REDINREN, Madrid, Spain; ${ }^{5}$ Institute of Pathology and Dept. of Nephrology, RWTH University of Aachen, Aachen, Germany
\end{abstract}

\subsection{6/lupus-2020-eurolupus. 178}

Background Most treatments reported to favourably impact on experimental lupus nephritis failed to reproduce these results in multicenter randomized controlled trials (RCT) in patients. Preclinical multicenter RCT (pRCT) may help to close the gap between preclinical and clinical trials. We therefore performed the first pRCT in lupus nephritis. We selected the Jak1/2 inhibitor baricitinib as a therapeutic intervention given that similar Jak/Stat inhibitors have shown protective effects in single center animal studies and baricitinib is currently tested in several clinical trials recruiting patients with systemic lupus (NCT 03843125, 03616964, 03616912).

Methods The effect of baricitinib was tested in a randomized, controlled, blinded pre-set study design at two Spanish (Madrid, Barcelona) and two German (Munich, Freiburg) academic sites. Each site included MRL/lpr mice of their own breeding colonies or from diverse commercial providers and kept at housing conditions as per their local standard operating procedures. Group size calculation was based on the assumption that baricitinib would reduce the primary endpoint, i.e. protein/creatinine ratio, by $20 \%$, with a type I error of 0.05 , type II error of 0.2 , and a power of 0.8 . Eligibility criteria were: female, 13-14 weeks old, had developed signs of systemic lupus erythematosus, had stress scores of less than 2 , and had no visible tumor or signs of infection. Block randomization was used to randomly assign mice at a $1: 1$ ratio to receive either $20 \mathrm{mg} / \mathrm{kg}$ baricitinib in $0.5 \%$ methylcellulose or vehicle daily by oral gavage for 4 weeks. Medication was provided in a blinded fashion by the coordinating study center. Periodically, each site collected urine and blood samples, 
and scored urine proteinuria, skin lesions and lymphadenopathy. At the end of the treatment interval, kidney, lung, spleen, skin, plasma and urine samples were collected and shipped to the coordinating study center for blinded analysis. All histopathological assessments were performed by an independent kidney pathology institute (RWTH Aachen, Germany) in a blinded fashion. The pre-set primary endpoint was urinary protein/creatinine ratio. Secondary endpoint analyses included plasma auto-antibodies levels, kidney histologic scores (activity and chronicity indices). Glomerular filtration rate and flow cytometry of splenocytes was performed on subgroups of mice at single centers.

Results A total of 56 mice were used, of which 13 in Madrid, 15 in Barcelona, 14 in Freiburg, and 14 in Munich. Mice were randomly assigned to baricitinib treatment $(n=28)$ or vehicle $(n=28)$. At treatment initiation, the average score of proteinuria (tested by sticks 0-4), skin lesion (0-4), and lymphadenopathy (0-6) for baricitinib group were $1.38 \pm 0.99$, $0.95 \pm 1.28$, and $2.10 \pm 1.80$, respectively; above scores for vehicle group were $1.45 \pm 1.10,0.77 \pm 1.02$, and $1.81 \pm$ 2.02 , respectively. Data analysis is ongoing and will be presented at the conference.

Conclusions Preclinical double-blind, randomized, controlled, multicenter trials are a novel tool in preclinical drug testing that might help to predict the outcome of randomized clinical trials.

\section{P135 A PHASE I, FIRST-IN-HUMAN STUDY TO ASSESS THE SAFETY, PHARMACOKINETICS AND PHARMACODYNAMICS OF SINGLE AND MULTIPLE ASCENDING DOSES OF M5049, A DUAL ANTAGONIST OF TLR7/8, IN HEALTHY SUBJECTS}

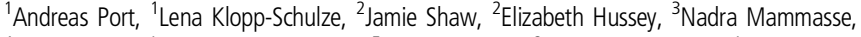
${ }^{4}$ Ying Zhang, ${ }^{1}$ Angelika Bachmann, ${ }^{5}$ Christian Reh, ${ }^{2}$ Kosalaram Goteti. ${ }^{1}$ Translational Medicine, Merck KGaA, Darmstadt, Germany; ${ }^{2}$ Translational Medicine; ${ }^{3}$ Biostatistics, Cytel, Paris, France; ${ }^{4}$ Global Patient Safety, EMD Serono Research and Development Institute*, Billerica, USA; ${ }^{5}$ Nuvisan GmbH, Neu-Ulm, Germany; *A business of Merck KGA, Darmstadt, Germany
\end{abstract}

\subsection{6/lupus-2020-eurolupus. 179}

Background Toll-like receptors 7 and 8 (TLR7/8) are widely expressed in a variety of immune cells and detect singlestranded RNAs, activating signalling cascades which trigger an immune response. Aberrant TLR7/8 activation by RNA-containing autoantibodies can result in autoimmunity. M5049 is an oral small molecule TLR7/8 antagonist which inhibits TLR7/8 activity in vitro and ex vivo and has demonstrated efficacy in mouse SLE models, suggesting potential to inhibit pathological immune complex activities in SLE patients.

Methods This was a phase I, randomized, double-blind, placebo-controlled (3:1), single and multiple ascending dose (SAD and MAD) study of oral M5049 conducted in healthy participants. SAD cohorts received a single dose of M5049 (1, 3, 9, 25, 50, 100 and $200 \mathrm{mg}$ ) or placebo, and MAD cohorts received M5049 (9, 25, $200 \mathrm{mg}$ once daily, 25 and $50 \mathrm{mg}$ twice daily) or placebo for 14 days. A sentinel dosing strategy was used in SAD cohorts. The study assessed safety, tolerability, pharmacokinetics (PK), and pharmacodynamics (ex vivostimulated cytokine secretion). Food effect was assessed in the 25 mg SAD cohort.

Results Preliminary results showed that M5049 was well-tolerated over the dosing interval, with no significant or doselimiting adverse events observed to date. PK parameters were linear and dose-proportional from 1 to $200 \mathrm{mg}$, with higher clearance and shorter half-life than predicted based on preclinical studies. Exposure-dependent inhibition of $e x$ vivo-stimulated IL-6 secretion was observed, with maximum inhibition achieved at $200 \mathrm{mg}$. PK results indicate a slight food effect.

Conclusions M5049 was well-tolerated with no safety signals in healthy participants, warranting further investigation as a potential treatment for autoimmune diseases, such as SLE.

\section{P136 SLEDAI RESPONSE PREDICTION TO BELIMUMAB THERAPY BY BASELINE LEVELS OF BLYS, APRIL AND CD8+ EFFECTOR MEMORY T-CELLS}

${ }^{1}$ Francesca Regola, ' Silvia Piantoni, ${ }^{1}$ Laura Andreoli, ${ }^{2}$ Torsten Lowin, ${ }^{1}$ Paolo Airò, ${ }^{1}$ Franco Franceschini, ${ }^{1}$ Angela Tincani, ${ }^{2}$ Georg Pongratz. ${ }^{1}$ Rheumatology and Clinical Immunology, ASST Spedali Civili, University of Brescia, Brescia, Italy; ${ }^{2}$ Rheumatology and Hiller Research Center for Rheumatology, University Hospital Düsseldorf, Düsseldorf, Germany

\subsection{6/lupus-2020-eurolupus. 180}

Background Systemic Lupus Erythematosus (SLE) patients show high levels of BLyS (B-lymphocyte stimulator) and other cytokines belonging to the tumor necrosis factor (TNF) superfamily ${ }^{1}$. Belimumab, a monoclonal antibody against BLyS, mainly targets B-cells ${ }^{2}$, but a BLyS-dependent T-cell activation pathway has been demonstrated ${ }^{3}$. Clinical studies showed that anti-DNA antibodies and complement levels at baseline are predictors of response to Belimumab. Our study aims at identifying other biomarkers as response predictors.

Methods Twenty-one SLE patients received Belimumab. Biomarkers belonging to the TNF superfamily (BLyS, APRIL, sBCMA, sCD40L, sTACI, TWEAK) were tested by ELISA in all patients and lymphocyte immunophenotyping was performed by flow cytometry in ten subjects at baseline and every six months. SLE-disease activity was assessed by SLEDAI-2K score.

Results BLyS and APRIL baseline serum levels and the number of CD3+CD8 + effector memory T-cells were correlated positively with SLEDAI-2K improvement after 12 months of treatment (Pearson correlation $=0.535 \quad(p=0.015), \quad 0.504$ $(\mathrm{p}=0.023)$ and $0.654(\mathrm{p}=0.040))$. After backwards exclusion from linear regression analysis including SLEDAI-2K, effector T-cell relative number and BLyS or APRIL at baseline, only APRIL remained as significant independent predictor of SLEDAI-2K improvement after 12 months (adjusted R square $=0.649 ; \mathrm{p}=0.025)$. After comparing TNF-family members levels and SLEDAI-2K at baseline, only BLyS showed the best predictive value (adjusted $\mathrm{R} 0.564$, $\mathrm{p}<0.001)$.

Conclusions In our cohort of SLE patients, baseline level of APRIL together with percentage of CD3+CD8+ effector memory T-cells, or BLyS serum level alone resulted as best predictors of response to Belimumab. Considering that immunophenotyping is rarely done in clinical practice, BLyS baseline serum levels alone could be used routinely as a good predictor of response, as suggested by post-hoc analyses of the BLISS study.

\section{REFERENCES}

1. $\mathrm{Ng} \mathrm{LG}$, et al. J Immunol, 2004.

2. Ramsköld D, et al. EBioMedicine, 2019.

3. Samy $\mathrm{E}$, et al. Int Rev Immunol, 2017. 